\title{
A PRELIMINARY COMPARISON OF GAS CORE FISSION AND INERTIAL FUSION FOR THE SPACE EXPLORATION INITIATIVE
}

\author{
Terry Kammash and David L. Galbraith \\ Department of Nuclear Engineering \\ The University of Michigan \\ Ann Arbor, MI 48109
}

(313) 764-0205

\begin{abstract}
Potential utilization of fission and fusion-based propulsion systems for solar system exploration is examined using a Mars mission as basis. One system employs the open cycle gas core fission reactor (GCR) as the energy source, while the other uses the fusion energy produced in an inertial confinement scheme known as the Magnetically Insulated Inertial Confinement Fusion (MICF) concept, to convert thermal energy into thrust. It is shown that while travel time for each approach may be comparable, the GCR must overcome serious problems associated with turbulent mixing, fueling and startup, among others, while the fusion approach must find ways to reduce the driver energy required for ignition.
\end{abstract}

\section{INTRODUCTION}

The Space Exploration Initiative calls for, among other things, a manned mission to the planet Mars sometime in the early part of the next century. Since space travel is hazardous and man is unable to endure long journeys without experiencing physical and mental degradation, it is imperative that such missions be completed in the shortest possible time. This in turn means that one or more "advanced" rocket propulsion schemes must be developed to meet these objectives. Two promising approaches in this regard are the open cycle gas core (Ragsdale 1990) fission reactor (GCR) and an inertial confinement fusion scheme known as the Magnetically Insulated Inertial Fusion (MICF) concept (Hasegawa et al. 1986). The principle of operation in GCR involves a critical uranium core in the form of a gaseous plasma that heats, through radiation, a hydrogen propellant which exits through a nozzle, thereby converting thermal energy into thrust as demonstrated in Figure 1. The MICF is a fusion scheme that combines the favorable aspects of inertial and magnetic fusions into one where physical confinement of the plasma is provided by a metal wall, while its thermal energy is insulated from that wall by a strong selfgenerated magnetic field, as illustrated in Figure 2.

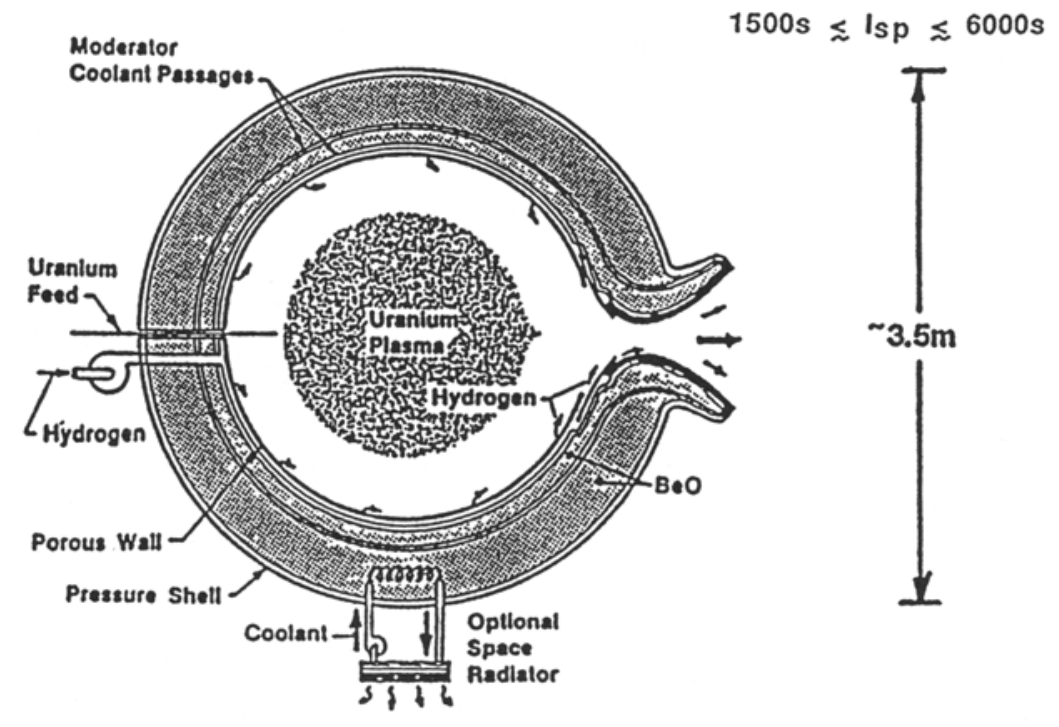

FIGURE 1. High Specific Impulse, Porous Wall Gas Core Engine (Courtesy of NASA, Lewis Research Center). 
In contrast to solid core reactors where temperature limitations, imposed by material melting, place severe constraints on rocket performance, the gas core concept circumvents these limitations because the nuclear fuel is allowed to exist in a high temperature $(10,000-100,000 \mathrm{~K})$ partially ionized state commonly referred to as the uranium plasma. Nuclear heat released as thermal radiation from the surface is absorbed by a surrounding envelope of seeded hydrogen propellant which is then expanded through a nozzle to generate thrust. With this scheme specific impulses of several thousand seconds appear to be feasible (Ragsdale 1990).

By comparison, the deuterium-tritium (DT) fusion plasma in MICF is generated inside a metal shell through ablation by an incident laser beam that enters the target through a hole as depicted in Figure 2. In addition to creating the plasma, the laser beam gives rise to an instantaneous, strong magnetic field which serves as a thermal insulator of the metal shell from the hot plasma during the fusion burn. The burn time in MICF is about one tenth of a microsecond (Kammash and Galbraith 1989) at the end of which a hot plasma consisting of the DT and metal shell particles is exhausted through a magnetic nozzle to produce the propulsive thrust. This approach to propulsion is also capable of producing specific impulses of several thousand seconds as will be noted shortly.

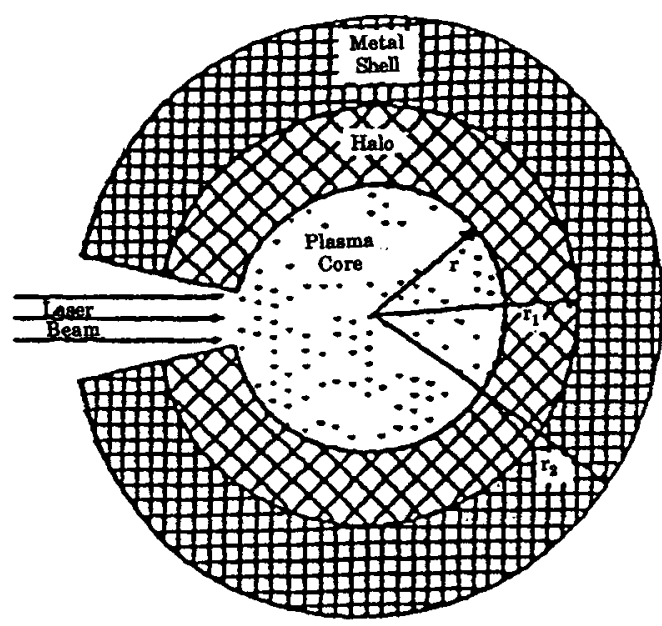

(a)

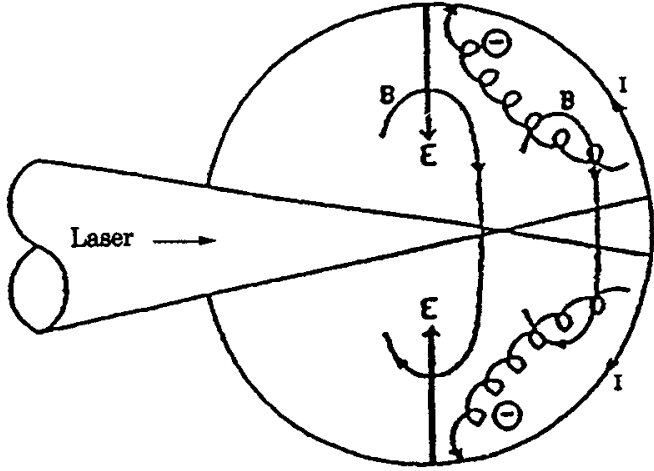

(b)

FIGURE 2. Schematic Diagram of (a) Plasma Formation and (b) Magnetic Field Formation in MICF.

\section{MAIOR PHYSICS AND ENGINEERING ISSUES}

While possessing unique propulsion capabilities, each of the above approaches must overcome some serious technological obstacles to make them suitable for space exploration. To highlight some of these issues we choose in each case a preliminary design for which relevant parameters are available. In the case of the open cycle gas core reactor we select a $7500 \mathrm{MW}$ design (Borowski 1987) in which the radius of the uranium core is 1 meter, the pressure in the system is $1000 \mathrm{~atm}$; and the hydrogen temperature is about $17,500 \mathrm{~K}$ which suggests that the fuel temperature is about 35,000 K (Kascak and Easely 1968). Our preliminary analysis of this system (Poston and Kammash 1992) shows that the mean velocity of the propellant which is commensurate with a cited mass flow rate of $4.5 \mathrm{~kg} / \mathrm{s}$ is about $5 \mathrm{~m} / \mathrm{s}$. The mean velocity of the uranium in the core is generally taken to be 10-15 times smaller than that of the propellant, and as a result it could be considered stationary. It is a known fact that when a fluid of density $\rho_{2}$ moves with velocity $v_{2}$ past another fluid of density $\rho_{1}$, which is stationary, in the presence of a gravitational force, the (sharp) boundary between them will, upon perturbation, undergo oscillations which under 
certain conditions can become unstable. Known as the Kelvin-Helmholtz instability (Chandrasekhar 1961) the sufficient condition for the occurrence of this instability is given by:

$$
\mathrm{v}_{2}^{2}>\frac{\mathrm{g} \rho_{1}}{\mathrm{k} \rho_{2}}
$$

where $\mathrm{g}$ is the gravitational acceleration (associated with flow curvature) and $\mathrm{k}$ is the wave number of the oscillation taken along the boundary that separates the two regions. In writing the above equation we made use of the fact that the uranium density $\rho_{1}$ for the conditions at hand is much larger than that of the propellant, $\rho_{2}$. The growth rate $\gamma$ of the instability may be expressed as

$$
\gamma=\frac{g}{v_{2}} \sqrt{\frac{\rho_{1}}{\rho_{2}}}
$$

which, along with Equation (1) allows us to calculate the diffusion coefficient and the particle flux across the spherical surface of the uranium core. At a pressure of $1000 \mathrm{~atm}$, the hydrogen and uranium temperatures cited above, and a core radius of 1 meter, the Kelvin-Helmholtz instability results in a loss of about $10 \mathrm{~kg} / \mathrm{s}$ of uranium which is unacceptably high and much larger than the $1 \%$ of hydrogen mass flow rate $(45 \mathrm{~g} / \mathrm{s})$ often cited as the loss due to turbulent mixing in gas core reactors. To see how such a loss impacts the travel time, we apply these results to a Mars mission for which we will consider a continuous burn acceleration/deceleration trajectory profile that assumes constant specific impulse ( $\mathrm{I}_{\text {sp }}$ ) and thrust (F). Using a dry vehicle mass of $123 \mathrm{Mg}$ as specified in the design cited earlier, we calculate the round trip times for various ratios of uranium mass flowrate $\left(\dot{\mathrm{m}}_{\mathrm{u}}\right)$ to hydrogen mass flowrate $\left(\dot{\mathrm{m}}_{\mathrm{H}}=4.5 \mathrm{~kg} / \mathrm{s}\right)$. The results are given in Table 1 .

TABLE 1. Effect of Turbulent Mixing on Travel Time.

\begin{tabular}{lccc}
\hline$\dot{\mathrm{m}}_{\mathrm{u}} / \dot{\mathrm{m}}_{\mathrm{H}}$ & $\mathrm{F}(\mathrm{KN})$ & $\mathrm{I}_{\mathrm{sp}}(\mathrm{s})$ & $\tau_{\mathrm{RT}}$ (days) \\
\hline & & & \\
0 & 87.6 & 1987 & 197 \\
0.01 & 87.7 & 1970 & 198 \\
0.1 & 88.5 & 1820 & 213 \\
0.5 & 92.2 & 1390 & 344 \\
1.0 & 96.8 & 1098 & 398 \\
2.0 & 106.02 & 940 & \\
\hline
\end{tabular}

These results are to be contrasted with those produced by the MICF fusion propulsion system (Kammash and Galbraith 1991) summarized in Table 2. 
TABLE 2. MICF Propulsion Capabilities.

Specific Impulse, $\mathrm{I}_{\mathrm{Sp}}$
Thrust (at 100 rep rate) $\mathrm{F}$
Laser Input Energy
Energy Gain Factor
Vehicle Dry Mass
Round trip time to Mars $\left(\tau_{\mathrm{RT}}\right)$

$4.51 \times 10^{3} \mathrm{~s}$

$41.2 \mathrm{KN}$

$2.59 \mathrm{MJ}$

724

$664 \mathrm{mT}$

138 days

The loss of uranium due to turbulent mixing and burn up requires fueling the core at a rapid rate in order to maintain criticality. We propose fueling by means of pellet injection. Noting that the ionization potential " $\varepsilon$ " of uranium is $6.18 \mathrm{eV}$, we can estimate the pellet ablation time $\mathrm{t}_{\mathrm{A}}$ from

$$
t_{A}=\frac{r_{p} n_{s} \varepsilon}{q_{e}}
$$

where $r_{p}$ is the radius of the injected pellet, $n_{S}$ is the solid state density, and $q_{e}$ is the heat flux, which in the case of uranium plasma at $35,000 \mathrm{~K}$, has a value of $6.2 \times 10^{28} \mathrm{eV} / \mathrm{cm}^{2}$ - $\mathrm{s}$. For a pellet of $0.5 \mathrm{~cm}$ radius whose mass is about $10 \mathrm{~g}$, the ablation time is about 1.5 microseconds, and the injection velocity needed to reach the center of the core would be about $670 \mathrm{~km} / \mathrm{s}$. This is a very high speed indeed and is perhaps out of reach for current or near term technology. But it should not be taken too seriously since a "bare" pellet does not remain bare once it enters the hot uranium core. Depending on what processes are postulated to take place in the "halo" region, a survival enhancement of the pellet of several hundred is possible and that may reduce the injection speed to several kilometers per second which puts it perhaps well within reach of technology in the not too distant future. Since the uranium burnup rate for the $7500 \mathrm{MW}$ reactor under consideration is quite small $(\sim 0.1 \mathrm{~g} / \mathrm{s})$ it is clear that an injection rate of about 1000 per second of such pellets will be required to make up for the loss of uranium due to the turbulent mixing phenomenon described earlier. Once again such an injection rate may be truly formidable, and the force needed to accelerate such particles to the desired velocity over a reasonable distance may be equally as challenging!

Although two or three major problems for GCR have been briefly highlighted in this paper, there are several others that are equally as critical but will not be addressed due to space limitations. These include the question of uranium confinement hydrodynamically or by magnetic fields, thermal and plasma instabilities, and questions associated with startup, shutdown and control. In the case of MICF, the major problem centers around target designs and fuel composition that would allow ignition at modest input laser energies.

\section{CONCLUSION}

Preliminary designs of a gas core fission reactor and an inertial fusion system have been examined in terms of their propulsive capability for a round trip mission to Mars. It is shown, on the basis of present day understanding of these approaches, that while trip times may be comparable, the fission system has many serious physics and engineering issues to overcome, while the fusion system, though better understood, must find means to reduce the input laser energy needed for large energy multiplication, and correspondingly a significant reduction in the dry weight of the vehicle. 


\section{Acknowledgments}

This work was carried out under the auspices of the Nuclear Engineering Department of the University of Michigan.

\section{Beferences}

Borowski, S. K. (1987) "Nuclear Propulsion-A Vital Technology for the Exploration of Mars and the Planets Beyond," NASA Technical Memorandum, 101 354, NASA Lewis Research Center, Cleveland, OH, 18 July 1987.

Chandrasekhar, S. (1961) Hydrodynamic and Hydromagnetic Stability, Dover Publications, N.Y.

Hasegawa, A., et al. (1986) "Magnetically Insulated Inertial Fusion: A New Approach to Controlled Thermonuclear Fusion," Phys. Rev. Letters, 56:139.

Kammash, T. and D. L. Galbraith (1989) "A High Gain Fusion Reactor Based on the Magnetically Insulated Inertial Confinement Fusion Concept," Nuclear Fusion 29:1079.

Kammash, T. and D. L. Galbraith (1991) "An Inertial Fusion Propulsion Scheme for Solar System Exploration," Proc. Eighth Symposium on Space Nuclear Power Systems, CONF-910116, Albuquerque, NM, 6-9 January 1991.

Kascak, A. F. and Easely, A. J. (1968) "Effect of Turbulent Mixing on Average Fuel Temperatures in a Gas-Core Nuclear Rocket Engine," NASA TN D-4882, NASA Lewis Research Center, Cleveland, OH, 6 August 1968.

Poston, D. I. and T. Kammash (1992) "Heat Transfer Model for an Open-Cycle Gas Core Nuclear Rocket," Ninth Symposium on Space Nuclear Power Systems, CONF-920104, held in Albuquerque, NM, 12-15 January 1992.

Ragsdale, R. G. (1990) Nuclear Thermal Propulsion, A Joint NASA/DOE/DOD Workshop, Cleveland, OH, 10-12 July 1990. 